\title{
Cervical myelopathy due to nuclear herniations in young adults: clinical and radiological profile, results of microdiscectomy without interbody fusion
}

\author{
B M Selladurai
}

\begin{abstract}
A study was made of the clinical and radiological characteristics and the results of microsurgical discectomy without interbody fusion, of 26 young adults, who presented with cervical myelopathy due to nuclear herniations. Neck trauma was not a significant aetiological factor. The disease produced moderate to very severe functional disability in most patients $(73 \%)$, in a relatively short period (mean symptom duration 6.3 months). Radiological assessment revealed the presence of canal stenosis, significant disc protrusions with paucity of spondylotic changes in most patients. At operation, soft disc lesions were found in $85 \%$ and sequestrated discs in $31 \%$. Microsurgical discectomy without fusion produced gratifying recovery of functional disability without significant deleterious effects on the cervical spine.
\end{abstract}

The classic descriptions of myelopathy due to cervical disc degeneration by Brain, ${ }^{1}$ and Brain $e t a l^{2}$ identify two distinct types of causative lesions: 1) nuclear herniation-where a circumscribed mass of disc material is extruded through a tear in the annulus fibrosus, most commonly occurring in young individuals and 2) annular protrusion-which is the more common lesion occurring in later life, where a collapsed, degenerate disc bulges the annulus. This bulge as well as adjacent osteophytes form the ridges typically seen in cervical spondylosis.

Few reports, however, ${ }^{3-7}$ have described cervical myelopathy due to nuclear herniation as a distinct clinicopathological entity. Some ${ }^{8-10}$ referred to these lesions as soft central discs. A majority of these studies included a significant proportion of patients of an older age group. In the study by $\mathrm{O}^{\prime}$ Connell ${ }^{5}$ the age range was from $22-53$ years, mean age 40 years. The age range was from $22-71$ years, mean age 47 years, in the study by O'Laoire and Thomas. ${ }^{7}$

In a previous report ${ }^{11}$ of a small series of patients, below 40 years of age, who presented with myelopathy due to nuclear herniations, discectomy under microscopic vision, without interbody fusion produced very gratifying results. The aims of this study are: 1) to define the clinical and radiological expressions of cervical myelopathy due to nuclear herniations in young adults (below 40 years); 2) to determine the immediate and long term results of microsurgical discectomy without interbody fusion; 3) to evaluate the effects of this procedure on the integrity of the cervical spine in this age group.

Restricting this study to young adults is aimed at eliminating any effects of coexisting spondylotic lesions, which have been reported in older patients with nuclear herniations, ${ }^{79}$ on the clinical picture and the therapeutic outcome of nuclear herniations.

\section{Materials and methods}

Between January 1980 and January 1990, 26 patients below the age of 40 years, who presented with cervical myelopathy due to nuclear herniations, were treated by microsurgical anterior discectomy without interbody fusion. The criteria for inclusion in this study are indicated in table 1 . These patients comprised $32 \%$ of a total of 68 patients of all ages who had surgery for cervical myelopathy due to nuclear herniations. During the same period a total of 120 microsurgical anterior procedures were performed for neurological manifestations of cervical disc degeneration.

Preoperative clinical evaluation of these patients consisted of a complete neurological examination and assessment of the degree of disability using a disability grading system (table 2). Radiological evaluation consisted of anteroposterior, lateral, oblique and dynamic views of the cervical spine and positive contrast myelography.

A standard operative approach to the anterior cervical spine was used through a right sided skin crease incision. The relevent disc spaces were identified by intraoperative radiography. A rectangular window was made in the anterior annulus and disc material was removed from the anterior disc space using rongeurs and currettes. Removal of the rest of the disc was done under microscopic vision, aided on occasions by the cautious use of a disc space retractor. All disc material was removed up to the posterior longitudinal ligament, which was identified under high magnification as a glistening structure with vertically aligned

\section{Table 1 Inclusion criteria}

\begin{tabular}{ll}
\hline 1 & $\begin{array}{l}\text { Age below } 40 \text { years } \\
\text { Myelopathy of sufficent severity to interfere signifi- } \\
\text { cantly with occupation and/or daily life }\end{array}$ \\
3 & $\begin{array}{l}\text { Absence of significant spondylotic changes in plain } x \\
\text { rays of the cervical spine }\end{array}$ \\
4 & $\begin{array}{l}\text { Disc herniation of } 3 \mathrm{~mm} \text { or more demonstrated at } \\
\text { positive contrast myelography }\end{array}$
\end{tabular}

and in revised form 25 August 1991 Accepted 24 September
1991
Neurosurgical Unit, B M Selladurai Dr B M Selladurai, Department of Surgery, Hospital Universiti Sain Malaysia, 16150 Kubang Kerian, Kelantan, Malaysia. 
Table 2 Disability grading system

\begin{tabular}{ll}
\hline Grade & Criteria \\
\hline A (No disability) & $\begin{array}{c}\text { Can run, walk more than 1 kilometre, aware of mild abnormality } \\
\text { in lower limbs, can stand from a squatting position without } \\
\text { help. } \\
\text { Difficulty with running, can walk up to 400 metres, can stand } \\
\text { from a squatting position with help, no interference with } \\
\text { occupation. }\end{array}$ \\
C (Mon walk up to 100 metres, difficulty in standing from a squatting \\
position, independant, able to engage in light occupations. \\
D (Severe disability) \\
E (Very severe disability) \\
Bedridden, completely dependant.
\end{tabular}

fibres. Occasionally, there was a tear in this ligament with sequestration of disc fragments into the spinal canal. The opening in the ligament was then enlarged to ensure complete removal of sequestrated disc fragments. The ligament was not opened as a routine. The cartilaginous end plates of the adjacent vertebral bodies were left intact.

Patients were mobilised on the first postoperative day. A cervical collar was used only if there was neck pain. Neurological examination and disabliity grading were reassessed and dynamic views of the cervical spine were performed at the time of discharge of each patient from hospital. Long term follow up of these patients consisted of serial assessments of neurological deficits and disability grading. Dynamic views of the cervical spine were performed one year after surgery and at the last follow up assessment.

\section{Results}

a) Preoperative clinical evaluation

1) Age distribution-ages ranged from 27 to 39 years, with a mean age of 33.8 years.

2) Sex distribution-there were 19 males (73\%) and 7 females (27\%).

3) Associated phenomena. One patient had an episode of neck trauma three years before onset of symptoms. In 2 patients an episode of neck trauma heralded the onset of symptoms. In one patient an episode of trauma aggravated the disease in mid course.

In 2 patients the onset of symptoms was during pregnancy, in the second and third trimester respectively.

4) Clinical symptoms-see tables 3, 4 and 5.

5) Preoperative disability grading. In all patients the degree of disability was assessed using the disability grading system in table 2 . The distribution of disability grades was as follows:

Grade A (No disability) 0 pts

Grade B (Mild disability) 7 pts (27\%)

Grade C (Moderate disability) 5 pts (19\%)

Grade D (Severe disability) 10 pts (39\%)

Grade E (Very severe disability) 4 pts (15\%)

6) Clinical signs-see table 6.

b) Radiological evaluation

1) Degree of spondylotic changes. No spondylotic changes were evident in 19 patients $(73 \%)$, changes were mild in 6 patients $(23 \%)$ and moderate in 1 patient (4\%).

2) Vertebral subluxation. There was no evidence of subluxation in any patient.
Table 3 Clinical symptoms

\begin{tabular}{lc}
\hline a) Duration of symptoms & Number of patients \\
less than 1 month & $2(8 \%)$ \\
1 to 6 months & $15(58 \%)$ \\
6 months to 1 year & $5(19 \%)$ \\
more than 1 year & $4(15 \%)$ \\
\hline b) Progression of symptoms & Number of patients \\
relentless progression & $24(92 \%)$ \\
progression $\rightarrow$ static state & $2(8 \%)$ \\
\hline
\end{tabular}

Table 4 Clinical symptoms

\begin{tabular}{lc}
\hline c) Initial symptom \\
Nature of symptom & Number of patients \\
\hline Motor symptoms & 12 \\
Paraparesis & 2 \\
Monoparesis (leg) & 2 \\
Monoparesis (arm) & 1 \\
Quadriparesis & 3 \\
Sensory symptoms & 1 \\
$\quad$ Numbness of upper limbs & 1 \\
Numbness of lower limbs & \\
Numbness of all 4 limbs & \\
\hline
\end{tabular}

Table 5 Clinical symptoms

\begin{tabular}{ll}
\hline d) Pain & Number of patients \\
\hline No pain & $15(58 \%)$ \\
Neck pain & $6(23 \%)$ \\
Brachalgia & $3(1 \%)$ \\
Burning pain in legs & $1(4 \%)$ \\
Lhemitte's phenomenon & $1(4 \%)$ \\
\hline e) Sphinctor involvement & $6(23 \%)$ \\
Bladder symptoms & $2(8 \%)$ \\
Bowel involvement & \\
\hline
\end{tabular}

Table 6 Clinical signs

\begin{tabular}{ll}
\hline a) Clinical type of lesion & Number of patients \\
\hline Exclusively motor deficits & \\
in upper and lower limbs & $11(41 \%)$ \\
in lower limbs only & $6(24 \%)$ \\
Transverse cord lesion & $4(16 \%)$ \\
Brachalgia and cord syndrome & $2(8 \%)$ \\
Central cord syndrome & $2(8 \%)$ \\
Brown-Sequard syndrome & $1(4 \%)$ \\
\hline b) Nature of deficits in upper limbs & Number of patients \\
Lower motor deficits & $12(46 \%)$ \\
Proprioceptive loss & $8(32 \%)$ \\
\hline c) Degree of disability in upper limbs & Number of patients \\
Mild (minimal limitation of activity) & $8(32 \%)$ \\
Moderate(independent, limitation & $8(32 \%)$ \\
of skilled movements) & $4(16 \%)$ \\
Severe(dependant) & \\
\hline
\end{tabular}

3) Spinal canal stenosis. The sagittal diameter of the spinal canal was $13 \mathrm{~mm}$ or less in 19 patients $(73 \%)$.

4) Degree of disc prolapse. A disc prolapse of $3 \mathrm{~mm}$ or more was considered significant. A prolapse of 3-4 mm was found in 10 patients (40\%). A prolapse of $5 \mathrm{~mm}$ or more was found in 16 patients (60\%), (fig 1 ).

5) Number of levels. A single level prolapse was found in 17 patients, 2 levels in 8 patients and a 3 level prolapse in 1 patient.

6) Levels of disc prolapse. The $C 3 / 4$ disc space was involved in 12 instances, C4/5 space in 10 instances, $\mathrm{C} 5 / 6$ space in 13 instances and the C6/7 space in one instance. 


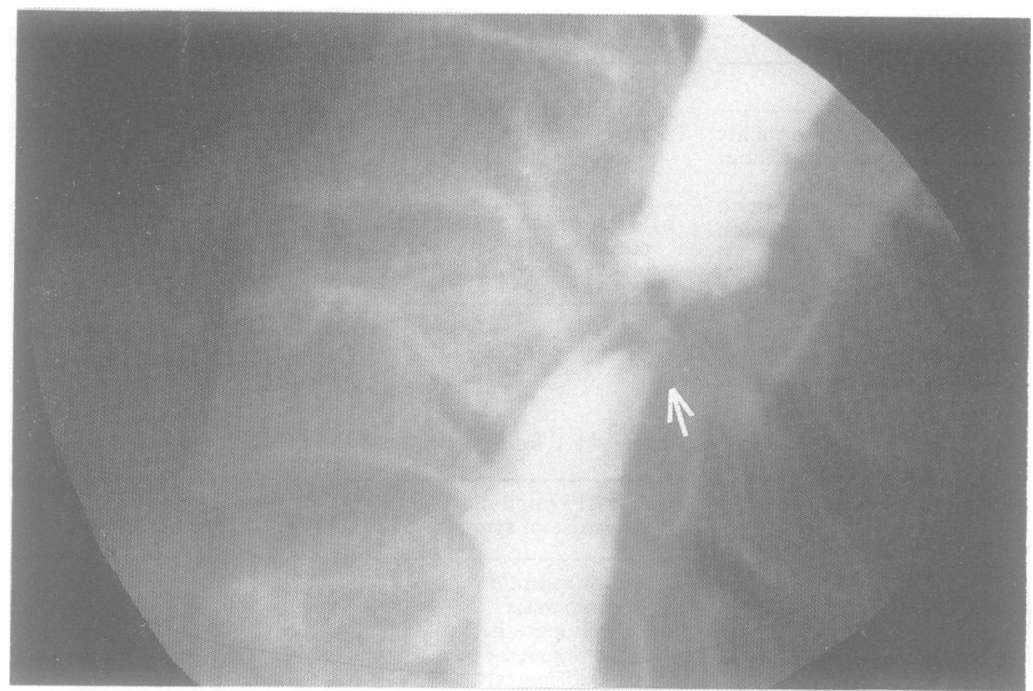

Figure 1 A magnified view ( $\times 2$ ) of a positive contrast myelogram showing a large (6 $\mathrm{mm}$ ) soft disc lesion (arrow). At surgery, the posterior longitudinal ligament was torn with sequestration of disc fragments. Note the evidence of canal stenosis and paucity of spondylotic changes.
Table 7 Postoperative complications

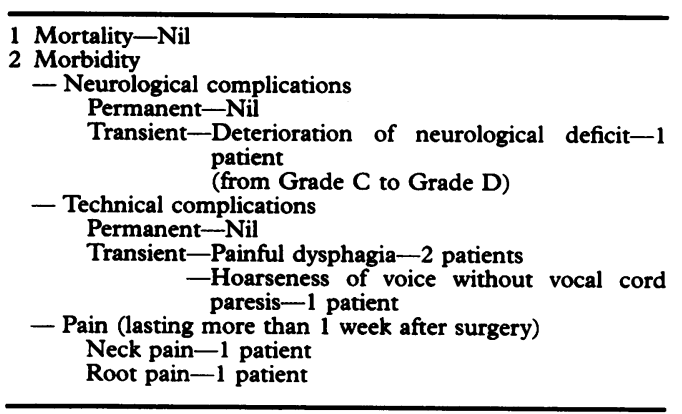

improved if the degree of disability had improved by at least one grade of assessment. Accordingly, 22 patients $(85 \%)$ showed improvement of disability (13 patients by 1 grade, 5 patients by 2 grades and 4 patients by 3 grades). Three patients remained in the preoperative grades of disability. One patient showed neurological deterioration immediately after surgery (from grade C to grade D) but gradually recovered to the preoperative grade at the time of discharge from hospital. Of the 14 patients in severely disabled grades $D$ and E, 6 showed improvement by 1 grade, 4 by 2 grades and 4 by 3 grades.

In view of the small number of patients with single level lesions no conclusions could be made as to whether the level of disc lesion influenced the degree of recovery. Lower motor deficits in the upper limbs improved in 6 out of 12 patients and proprioceptive loss improved in 5 out of 8 patients. Bladder symptoms improved in 4 out of 6 patients. Dynamic views of the cervical spine performed at the time of discharge from hospital did not show subluxation or collapse at any of the operated disc spaces, in all the patients.

\section{f) Long term follow up (see table 8)}

An up to date long term follow up was possible in 20 patients $(77 \%)$, from periods ranging

Table 8 Results of surgical treatment

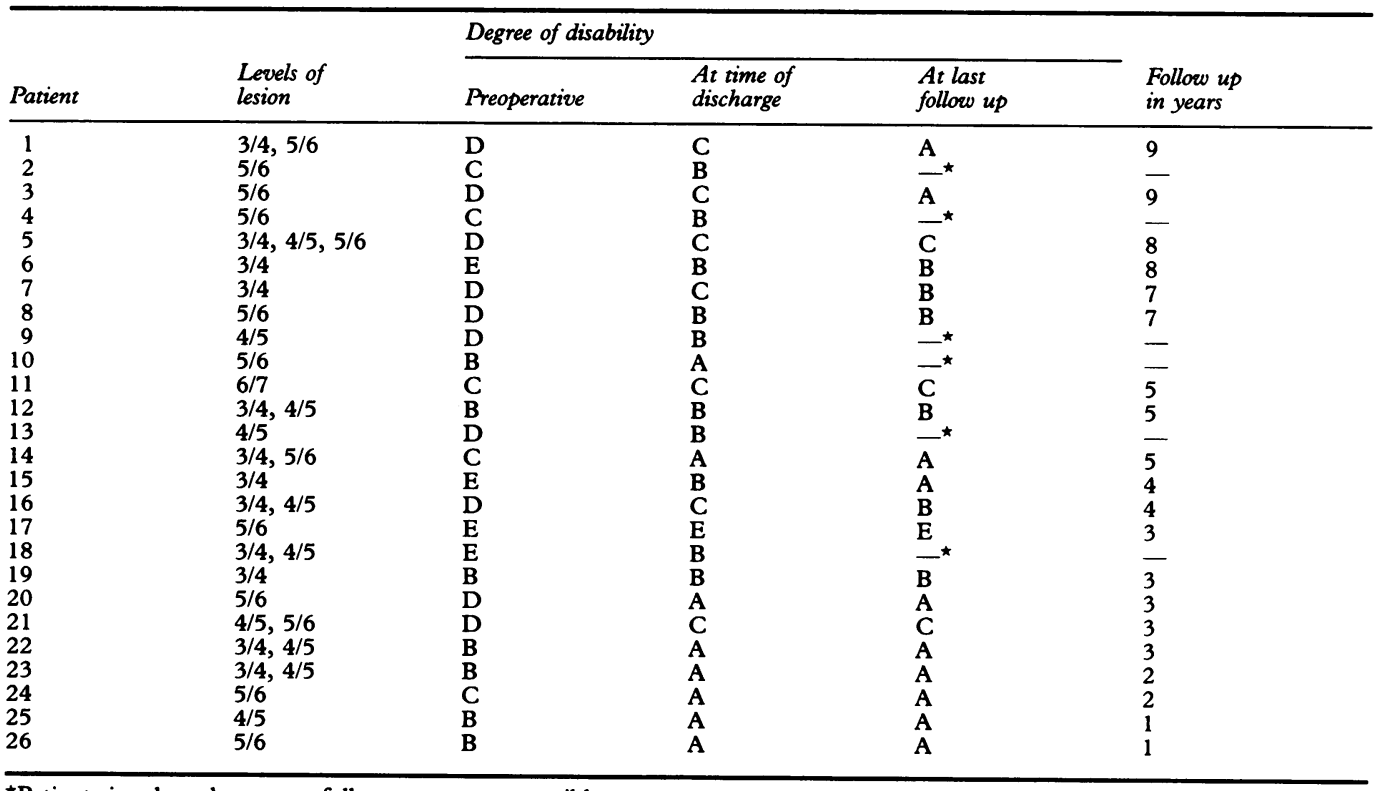

^Patients in whom long term follow up was not possible. 

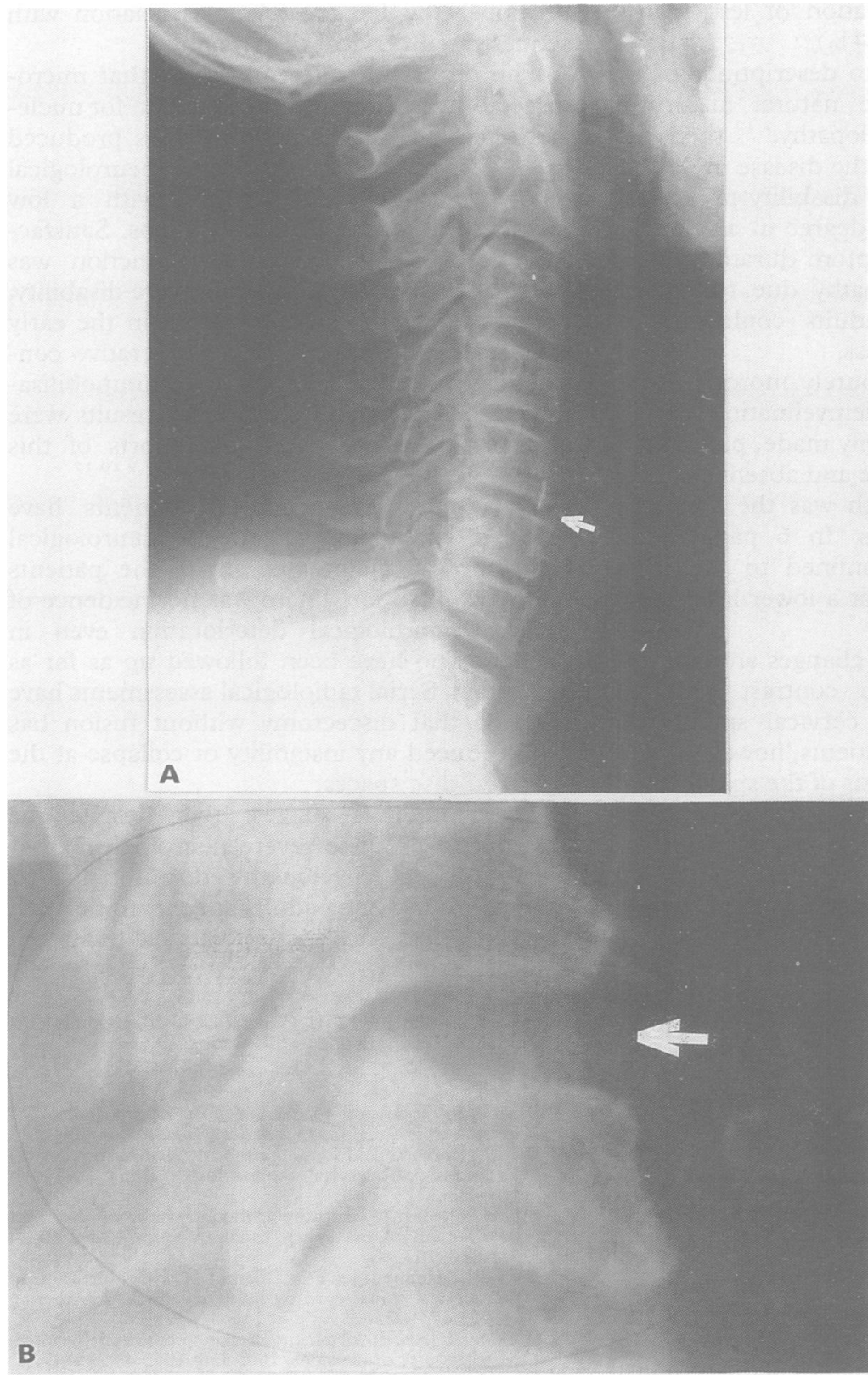

Figure 2 a) A lateral view of a cervical spine seven years after discectomy without fusion at the C5/6 space (arrow), showing no evidence of collapse or subluxation at the interspace; b) a magnified ( $\times 2$ ) lateral view of a C4/5 interspace, nine years after discectomy without fusion, showing no evidence of collapse, subluxation or significant spondylotic changes.

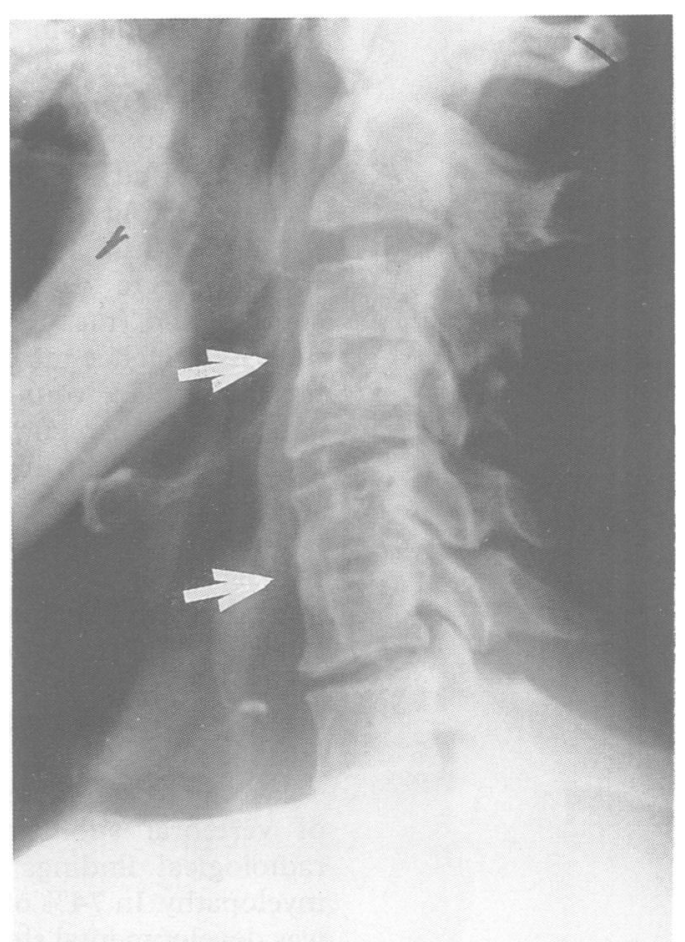

Figure 3 A lateral view of a cervical spine, five years afier discectomy without fusion at C3/4 and C5/6 spaces, showing spontaneous radiological fusion at the inter spaces (arrows). Note absence of subluxation at adjacent spaces.

dynamic views of the cervical spine revealed no evidence of subluxation or collapse at the operated disc spaces (fig 2). Seven patients showed radiological union at the sites of discectomy (fig 3). Three patients showed a mild increase of spondylotic changes in the cervical spine without accompanying symptoms.

\section{Discussion}

Previous studies of cervical myelopathy due to nuclear herniations, ${ }^{579}$ emphasised the need to distinguish these lesions from annular herniations associated with cervical spondylotic myelopathy. This distinction was especially relevent in view of the gratifying results of surgical treatment of nuclear herniations, if undertaken early in the course of the disease. In this study, cervical myelopathy due to nuclear herniations in all age groups $(22 \%$ of all cervical disc surgery) was of a higher incidence than in previous studies. ${ }^{8-10}$ The occurrence of nuclear herniations in young adults in this study (38\% of all patients with nuclear herniations) was similar to that reported in the study of O'Laoire and Thomas.

In the original descriptions of nuclear herniations in young adults, ${ }^{1}$ neck trauma was considered a common aetiological factor and the clinical presentation was one of painful, rapidly progressive cervical cord compression. By contrast, in this study neck trauma was associated with the onset of symptoms in only 3 patients $(12 \%)$, sixteen patients did not have neck or radicular pain. The presentation was 
dramatic (symptom duration of less than 1 week) in only 1 patient (4\%).

However, in contrast to descriptions of the clinical picture and the natural history of cervical spondylotic myelopathy, ${ }^{212}$ there was relentless progression of the disease in $92 \%$ of patients and in $73 \%$ the disability progressed to a moderate to severe degree in a relatively short period (mean symptom duration of 6.3 months). Hence myelopathy due to nuclear herniations in young adults could not be considered a benign illness.

In patients who had purely motor involvement, a diagnosis of demyelinating disease could have been mistakenly made, particularly in view of the younger age and absence of neck pain or brachalgia, which was the case in a majority of our patients. In 6 patients the abnormal signs were confined to the lower limbs and a cord lesion at a lower level could have been suspected.

Paucity of spondylotic changes and absence of vertebral subluxation contrast with the radiological findings of cervical spondylotic myelopathy. In $74 \%$ of patients, however, there was developmental stenosis of the spinal canal. This finding is compatible with observations made of the association between developmental canal stenosis and cervical spondylotic myelopathy. ${ }^{141516}$ As observed by O'Laoire and Thomas ${ }^{7}$ in their study of nuclear herniations, a higher incidence of lesions at the upper disc spaces was evident in our patients $(61 \%$ of disc lesions were at $\mathrm{C} 3 / 4$ and $\mathrm{C} 4 / 5$ spaces). However, in contrast to the findings of O'Connell ${ }^{5}$ who described only single level nuclear herniations, lesions were found at 2 levels in 8 patients and 3 levels in 1 patient.

The use of the operating microscope greatly enhanced the margin of safety of operative manoeuvres such as widening of an opening in the posterior longitudinal ligament or removal of a sequestrated disc fragment. Coaxial illumination, magnification and depth perception provided by this instrument enabled radical removal of disc material without the need to remove the adjacent margins of the vertebral bodies. The disc material removed from a majority of the patients in this study was macroscopically different from classic degenerate discs, raising the possibility that physicochemical and ultrastructural analysis of "soft" discs may reveal features that distinguish them from classic degenerate discs. Eight patients $(30 \%)$ had tears of the posterior longitudinal ligament with sequestration of free disc fragments into the spinal canal. Only one of these patients, however, presented with acute pain and a rapidly progressive cord lesion. The mean symptom duration in these 8 patients was 4.7 months. Thus ruptures of the posterior longitudinal ligament with sequestration of disc fragments do not necessesarily present in a catastrophic fashion. All 8 patients with sequestrated discs had large anterior indentations of the column of contrast $(5 \mathrm{~mm}$ or more), demonstrated at positive contrast myelography. This finding has been described previously by Isu et $a l^{13}$ in association with sequestrated cervical disc lesions.

The results of this study show that microdiscectomy without interbody fusion for nuclear herniations in young adults, has produced gratifying results, as shown by neurological recovery in $85 \%$ of patients, with a low incidence of technical complications. Satisfactory recovery of neurological function was possible even in patients with severe disability, provided surgery was carried out in the early stages of the disease. The postoperative convalescence was smooth and collar immobilisation was rarely required. Similar results were also the feature of previous reports of this procedure for nuclear herniations. ${ }^{791017}$

Long term follow up assessments have revealed that improvement of neurological function was maintained in all the patients followed up so far. There was no incidence of delayed neurological deterioration even in patients who have been followed up as far as nine years. Serial radiological assessments have revealed that discectomy without fusion has not produced any instability or collapse at the operated disc spaces.

These findings suggest that despite the capacity to produce severe neurological disability, cervical myelopathy due to nuclear herniations in young adults appears to be a self limiting illness once prompt surgical treatment is undertaken.

I am grateful to Dr J B Peiris, General Hospital, Colombo, Sri Lanka, for his contributions to this study.

1 Brain WR. Discussion on rupture of the intervertebral disc in the cervical region. Proc $R$ Soc Med 1948;41:509-2.

2 Brain WR, Northfield D, Wilkinson $M$. Neurological manifestations of cervical spondylosis. Brain 1952;75: 187-225.

3 Mixter WJ, Barr JS. Rupture of the intervertebral disc with involvement of the spinal canal. $N$ Engl $f$ Med 1934; 211:210-15.

4 Bucy PC, Heimburger FC, Oberhill HR. Compression of the cervical spinal cord by herniated discs. $\mathcal{f}$ Neurosurg 1948;5:471-92.

5 O'Connell JEA. Involvement of the spinal cord by intervertebral disc protrusions. Br $₹$ Surg 1955;43:225-47.

6 Campbell AMG, Phillips DG. Cervical disc lesions with Neurological disorder. BMF 1960;2:481-5.

7 O'Laoire SA, Thomas DGT. Spinal cord compression due to prolapse of intervertebral disc (herniation of nucleus pulposus). I Neurosurg 1983;57:847-53.

8 Scoville WB. Types of cervical disc lesions and their surgical approaches. $\tilde{f} A M A$ 1966;196:479-5.

9 Bertalanffy H, Eggert HR. Clinical and long term results of anterior discectomy without fusion for the treatment of cervical radiculopathy and myelopathy. Acta Neurochir (Wien) 1988;90:127-5.

10 Robertson JT. Anterior discectomy without fusion. In: S B Dunsker, ed. Cervical spondylosis. NY: Raven Press, 1981:181-90.

11 Selladurai BM, Peiris JB. Cervical myelopathy due to disc disease in the young. Ceylon Med F 1981;26:59-63.

12 Lees F, Turner JWA. Natural history and prognosis of cervical spondylosis. BMF 1963;2:1607-10.

13 Isu T, Iwasaki $Y$, Miyasaka $K$, Abe $H$, Tashiro $K$, Ito T. A reappraisal of the diagnosis in cervical disc disease: The posterior longitudinal ligament perforated or not. Neuroradiology 1986;28:215-20.

14 Payne J, Spillane J. The cervical spine. An anatomicopathological study of 70 specimens (using a special technique) with special reference to cervical spondylosis. Brain 1957;80:571-96.

15 Burrows EH. The saggital diameter of the spinal canal in cervical spondylosis. Clin Radiol 1963;14:77-86.

16 Nurick S. The pathogenesis of the spinal cord disorder associated with cervical spondylosis. Brain 1972;95: 87-100.

17 Husag $\mathrm{L}$, Probst Ch. Microsurgical anterior approach to cervical discs. Review of 60 consecutive cases of dis229-42. 